\title{
Visible light mediated aerobic oxidative hydroxylation of 2-oxindole-3-carboxylate esters: an alternative approach to 3-hydroxy-2-oxindoles
}

https://doi.org/10.1515/hc-2019-0114

Received June 04, 2020; accepted October 03, 2020.

Abstract: A convenient aerobic oxidative hydroxylation of 3-substituted oxindoles under mild reaction conditions is described herein. This process was accomplished by the activation of molecular oxygen in the air in the presence of a photocatalyst under the irradiation of visible light. The desired product was delivered in up to $89 \%$ yield without the addition of base or stoichiometric oxidant.

Keywords: Visible light, Photocatalysis, Oxindole, Aerobic oxidation

\section{Introduction}

The 3-substituted-3-hydroxy-2-oxindole framework is a privileged motif that is abundant in naturally occurring alkaloids and biologically active products [1-2]. For instance, Convolutamydine $\mathrm{A}$, which is isolated from the marine bryozoan Amathia convolute, was found to be a potent inhibitor in the differentiation of HL-60 human promyelocytic leukaemia cells [3]. Discovered in Hibiscus moscheutos L methyl 2-(3-hydroxy2-oxoindolin-3-yl)acetate acted as an anti-oxidant [4]. $(R)-(+)-3$-cyanomethyl-3-hydroxyoxindole from Rheum maximowiczii Losinsk (Polygonaceae) exhibited activation/inhibition of specific cytokines [5]. 3-Substituted3-hydroxy-2-oxindoles also act as synthetic precursors

\footnotetext{
*Corresponding author: Xu-Dong Xia, Engineering Laboratory of Chemical Resources Utilization in South Xinjiang of Xinjiang Production and Construction Corps, College of Life Sciences, Tarim University, Alar, Xinjiang 843300, P. R. China,

E-mail: xiaxudong_10@163.com

Jinge Wang, Siyitemer Osman, Xinjiang Lu and Junyi Chen, Engineering Laboratory of Chemical Resources Utilization in South Xinjiang of Xinjiang Production and Construction Corps, College of Life Sciences, Tarim University, Alar, Xinjiang 843300, P. R. China
}

in some significant transformations [6-9]. Consequently, their construction is of interest to organic chemists. Previously, several elegant methodologies were applied to build 3-substituted-3-hydroxy-2-oxindoles [10-12]. Transition metal catalyzed intramolecular cyclization of $\alpha$-keto aromatic amides was reported to synthesize these versatile building blocks [13-15], and direct addition to isatin derivatives was also an efficient approach [16-27]. Moreover, straightforward hydroxylation of 3-substituted-2-oxindoles was also accomplished [28-33]. However, milder, greener, and more efficient methods to change the substituent pattern of $\mathrm{C}-3$ on oxindole rings are still pursued.

Recently, many outstanding groups have sought to make use of light as a clean and abundant source of energy for chemical transformations [34-39]. Many reactions were carried out in the presence of oxygen under mild conditions. Furthermore, as an ideal oxidant molecular oxygen has already participated in the construction of a series of structurally diverse skeletons through photocatalysis [34-40]. For instance, Córdova accomplished the $\alpha$-hydroxylation of ketones and aldehydes through the utilization of molecular oxygen by photocatalysis [41-42]. In 2012, Meng and co-works documented a hydroxylation of $\beta$-oxo esters catalyzed by a phase transfer catalyst and tetraphenylporphine under the irradiation of a 100W halogen lamp, in which singlet oxygen took part [43]. Recently, Xiao's group designed and synthesized a new bifunctional visible light photocatalyst and used it in the asymmetric aerobic oxidation of $\beta$-ketoesters [44]. As we continue to be interested in photochemistry, in this communication, we developed an $\alpha$-hydroxylation of 3-carboxylate oxindole derivatives by means of photocatalysis under mild conditions. The reaction was carried out in air under the irradiation of a household light bulb. Compared with previous work, this work eliminates the need for addition of base and stoichiometric oxidant. We intended this work to further extend the scope of our previously developed research [45]. 


\section{Results and discussion}

Initially, the 3-substituted-2-oxindole (1a) was chosen as the model substrate and the reaction was first studied in the presence of commonly used photosensitizers. As shown in Table 1, all of the photocatalysts tested could successfully give the desired product in moderate yields in the presence of an $\mathrm{O}_{2}$ balloon under the irradiation of visible light (entries 1-4). Among them $\left[\operatorname{Ir}(\mathrm{ppy})_{2}(\mathrm{dtbbpy})\right] \mathrm{PF}_{6}$ proved to be the most promising for further optimization. To our delight, changing the oxygen source from an $\mathrm{O}_{2}$ balloon to atmospheric air did not significantly affect the hydroxylation efficiency (entry 2 vs entry 5). The desired product was still obtained in $70 \%$ yield. Control experiments showed that photocatalyst, visible light and $\mathrm{O}_{2}$ were indispensable for this transformation. No product was afforded when the reaction was conducted in the absence of any of these three parameters (entries 6-8). Subsequently, a variety of solvents were examined, and the choice of the reaction media was found to be essential for the hydroxylation. When using
$\left[\operatorname{Ir}(\mathrm{ppy})_{2}(\mathrm{dtbbpy})\right] \mathrm{PF}_{6}$, polar solvents such as DMF, DMSO, and $\mathrm{MeOH}$ showed relatively low compatibility compared with $\mathrm{MeCN}$, giving the product in yields ranging from 40\%-64\% (entries 9-11). Only a trace amount of $2 \mathbf{a}$ was detected when less polar solvents were employed (entries 12-14). Fortunately, we found that $\mathrm{CF}_{3} \mathrm{CH}_{2} \mathrm{OH}$ markedly improved the yield of this process to $88 \%$ (entry 15). Moreover, a shorter reaction time was observed when using silica gel as an additive (entry 16), possibly because the substrate was activated by H-bonding and/or the solubility of molecular oxygen was increased [46-47].

With the optimized conditions in hand, the study was then focused on extending the substrate scope. The electronic effect on the benzene ring of the oxindole moiety was first examined (Table 2, entries a-f). In general, substrates bearing an electron-withdrawing group provided better results than substrates with electrondonating groups. Specifically, when an electron-donating group such as methoxy (1c) was installed on the benzene ring, the reaction proceeded sluggishly, and the desired

Table 1 Screening the reaction conditions and control experiment ${ }^{a}$

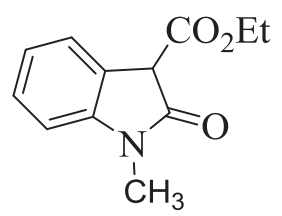

$1 \mathrm{a}$

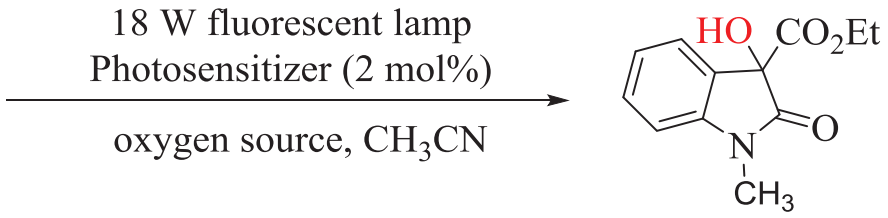

2a

\begin{tabular}{|c|c|c|c|c|c|}
\hline Entry & Photosensitizer & Solvent & Oxygen source & Time (h) & Yield $\%^{b}$ \\
\hline 1 & $\mathrm{Ru}(\mathrm{bpy})_{3} \mathrm{Cl}_{2} \cdot 6 \mathrm{H}_{2} \mathrm{O}$ & $\mathrm{CH}_{3} \mathrm{CN}$ & $\mathrm{O}_{2}$ balloon & 3 & 68 \\
\hline 2 & {$\left[\operatorname{lr}(\mathrm{ppy})_{2}(\mathrm{dtbbpy})\right] \mathrm{PF}_{6}$} & $\mathrm{CH}_{3} \mathrm{CN}$ & $\mathrm{O}_{2}$ balloon & 4 & 72 \\
\hline 3 & {$\left[\operatorname{Ir}\left(\mathrm{dF}\left(\mathrm{CF}_{3}\right) \mathrm{ppy}\right)_{2}(\mathrm{dtbbpy})\right] \mathrm{PF}_{6}$} & $\mathrm{CH}_{3} \mathrm{CN}$ & $\mathrm{O}_{2}$ balloon & 3 & 61 \\
\hline 4 & Eosin $Y$ & $\mathrm{CH}_{3} \mathrm{CN}$ & $\mathrm{O}_{2}$ balloon & 3 & 69 \\
\hline 5 & {$\left[\operatorname{lr}(\mathrm{ppy})_{2}(\mathrm{dtbbpy})\right] \mathrm{PF}_{6}$} & $\mathrm{CH}_{3} \mathrm{CN}$ & air & 4 & 70 \\
\hline 6 & Without photocatalyst & $\mathrm{CH}_{3} \mathrm{CN}$ & air & 4 & 0 \\
\hline $7^{c}$ & {$\left[\operatorname{lr}(\mathrm{ppy})_{2}(\mathrm{dtbbpy})\right] \mathrm{PF}_{6}$} & $\mathrm{CH}_{3} \mathrm{CN}$ & air & 4 & 0 \\
\hline $8^{d}$ & {$\left[\operatorname{lr}(\mathrm{ppy})_{2}(\mathrm{dtbbpy})\right] \mathrm{PF}_{6}$} & $\mathrm{CH}_{3} \mathrm{CN}$ & air & 4 & 0 \\
\hline 9 & {$\left[\operatorname{lr}(\mathrm{ppy})_{2}(\mathrm{dtbbpy})\right] \mathrm{PF}_{6}$} & DMF & air & 7 & 44 \\
\hline 10 & {$\left[\operatorname{lr}(\mathrm{ppy})_{2}(\mathrm{dtbbpy})\right] \mathrm{PF}_{6}$} & DMSO & air & 7 & 40 \\
\hline 11 & {$\left[\operatorname{lr}(\mathrm{ppy})_{2}(\mathrm{dtbbpy})\right] \mathrm{PF}_{6}$} & $\mathrm{CH}_{3} \mathrm{OH}$ & air & 4 & 64 \\
\hline 12 & {$\left[\operatorname{Ir}(\mathrm{ppy})_{2}(\mathrm{dtbbpy})\right] \mathrm{PF}_{6}$} & $\mathrm{CH}_{2} \mathrm{Cl}_{2}$ & air & 4 & trace \\
\hline 13 & {$\left[\operatorname{lr}(\mathrm{ppy})_{2}(\mathrm{dtbbpy})\right] \mathrm{PF}_{6}$} & THF & air & 4 & trace \\
\hline 14 & {$\left[\operatorname{lr}(\mathrm{ppy})_{2}(\mathrm{dtbbpy})\right] \mathrm{PF}_{6}$} & Toluene & air & 4 & trace \\
\hline 15 & {$\left[\operatorname{Ir}(\mathrm{ppy})_{2}(\mathrm{dtbbpy})\right] \mathrm{PF}_{6}$} & $\mathrm{CF}_{3} \mathrm{CH}_{2} \mathrm{OH}$ & air & 12 & 88 \\
\hline $16^{e}$ & {$\left[\operatorname{lr}(\mathrm{ppy})_{2}(\mathrm{dtbbpy})\right] \mathrm{PF}_{6}$} & $\mathrm{CF}_{3} \mathrm{CH}_{2} \mathrm{OH}$ & air & 10 & 88 \\
\hline
\end{tabular}

${ }^{a} \mathrm{~A}$ mixture of $1 \mathrm{a}(0.3 \mathrm{mmol})$ and photocatalysts $(2 \mathrm{~mol} \%)$ in solvent $(3 \mathrm{~mL}, 0.1 \mathrm{M})$ were stirred at room temperature under the irra-

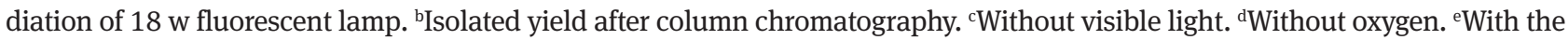
addition of silica gel (30 mg per $1 \mathrm{~mL}$ solvent). DMF: N,N-Dimethylformamide. DMSO: Dimethyl sulfoxide. THF: Tetrahydrofuran. 
Table 2 Substrate scope of the photocatalytic system ${ }^{a}$
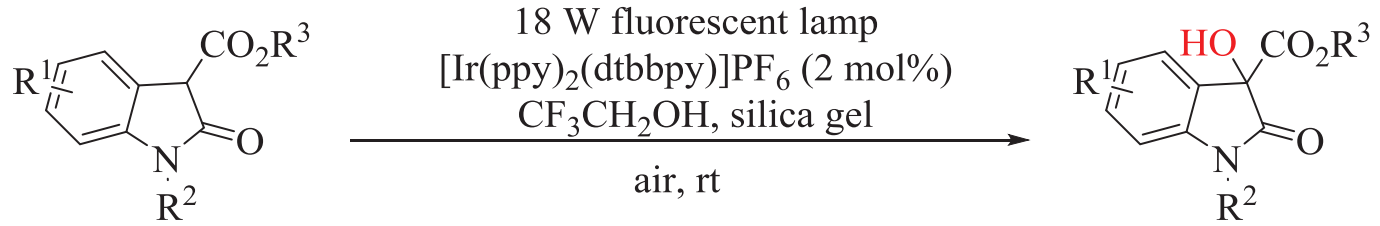

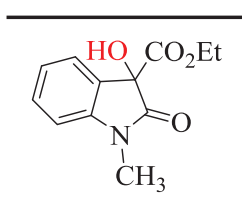

2a, $11 \mathrm{~h}$

$88 \%$ yield $^{b}$<smiles>CCOC(=O)C(O)(O)c1cc(Br)ccc1N(C)CC</smiles>

2f, $18 \mathrm{~h}$ $84 \%$ yield<smiles>CCOC(=O)C(O)(C(=O)Nc1ccccc1Br)C(=O)OCC</smiles>

2k, 33 h

$79 \%$ yield<smiles>CCOC(=O)c1cc(C)ccc1N(C)C(=O)OCC</smiles>

$2 \mathbf{b}, 48 \mathrm{~h}$

$77 \%$ yield<smiles>CCOC(=O)C1(O)C(=O)N(C)c2cc(Br)ccc21</smiles>

$2 \mathrm{~g}, 24 \mathrm{~h}$

$20 \%$ yield

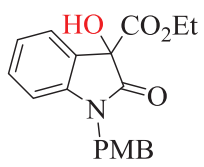

21, $34 \mathrm{~h}$

$74 \%$ yield

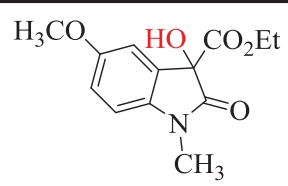

2c, $48 \mathrm{~h}$

$57 \%$ yield

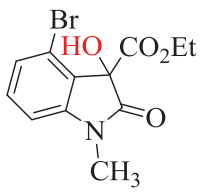

2h, 72 h

$0 \%$ yield

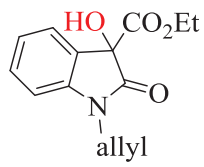

2m, 48 h

$39 \%$ yield

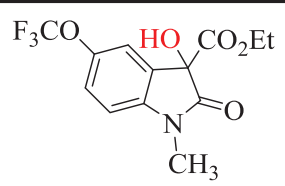

2d, $12 \mathrm{~h}$

$89 \%$ yield

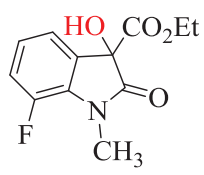

2i, 22 h

$79 \%$ yield

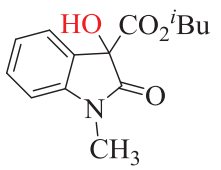

2n, 48 h

$77 \%$ yield

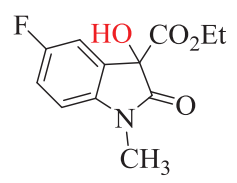

2e, $18 \mathrm{~h}$ $86 \%$ yield<smiles>CCOC(=O)C1(O)C(=O)Nc2ccccc21</smiles>

2j, 36 h $82 \%$ yield

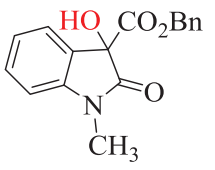

2o, 48 h

$62 \%$ yield

${ }^{\mathrm{a}} \mathrm{A}$ mixture of 1a-10 (0.5 mmol), [ $\left.\operatorname{Ir}(\mathrm{ppy})_{2}(\mathrm{dtbbpy})\right] \mathrm{PF}_{6}(2 \mathrm{~mol} \%)$, and silica gel (30 mg per $1 \mathrm{~mL}$ solvent) in solvent (5 mL, $\left.0.1 \mathrm{M}\right)$ were stirred at room temperature under the irradiation of an $18 \mathrm{w}$ fluorescent lamp open to air. ${ }^{\mathrm{b}}$ Isolated yield after column chromatography.

product was delivered in only 57\% yield after 2 days. Moreover, the substituted position of the $\mathrm{R}^{1}$ group had a big influence on the results of the hydroxylation. For example, 6-Br substituted oxindole carboxylate only gave $\mathbf{2 g}$ in $20 \%$ yield, while 4-Br substituted oxindole carboxylate (1h) did not react upon exposure to the optimized standard conditions, since $\mathbf{1 h}$ exists exclusively in its keto form. However, the 7-F substituted derivative (1i) was quite tolerated, and the hydroxylated product $\mathbf{2 i}$ was afforded in a yield of $79 \%$. Gratifyingly, when the N-protecting group was replaced with $\mathrm{H}, \mathrm{Bn}$ and $\mathrm{PMB}$, the reaction proceeded smoothly and afforded the desired products in moderate to good yields (entries $\mathbf{j}-1)$. In the case of $\mathbf{1 m}$, with an allyl as the protecting group, the product was obtained only in $39 \%$ yield. Finally, the ester moiety was also investigated. 1n and 10 were not fully hydroxylated even after 2 days. Under the standard reaction conditions, they afforded the products (2n and 2o) in 77\% and 62\% yields, respectively.

By changing the solvent to THF, the protocol could be extended to the synthesis of 3-hydroxy-3-phenyloxindole (2p) and 3-hydroxy-1-methyl-3-phenyloxindole (2q) derivatives in $60 \%$ and $17 \%$ yield, respectively (Scheme 1) [33]. Subsequently, in order to test the application of the protocol we increased the reaction scale to gram scale, as shown in Scheme 1. When the reaction ran on a larger scale, a decrease in reaction efficiency and in yield of the hydroxylated product was observed. After 48 hours the starting material could not be completely transferred to the product, and only $60 \%$ of the target molecule was obtained.

A plausible mechanism for this transformation is outlined in Scheme 2. Based on the previous work of Xiao's group, there was an equilibration of the substrate (1a) between its keto and enol form [45]. Visible light irradiation of the photocatalyst brought it to its excited state [Ir] ${ }^{\star}$, and oxidative quenching between $\mathbf{1} \mathbf{a}^{\prime}$ and $[\mathbf{I r}]^{\star}$ delivered the radical intermediate $\mathbf{A}$. The reduced photocatalyst [Ir] underwent a single electron transfer with oxygen to complete the photocatalytic cycle, to obtain an oxygen radical anion. Thereafter, intermediate $\mathbf{A}$ went through a radical coupling with the oxygen radical anion, giving rise 

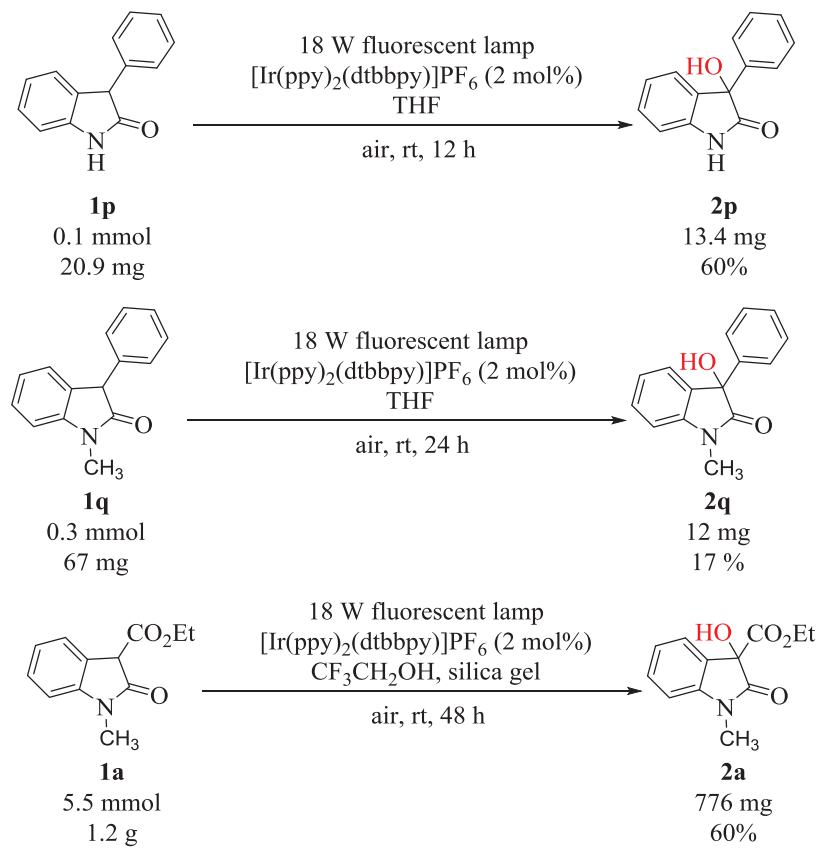

Scheme 1 Substrate scope extension and large scale reaction

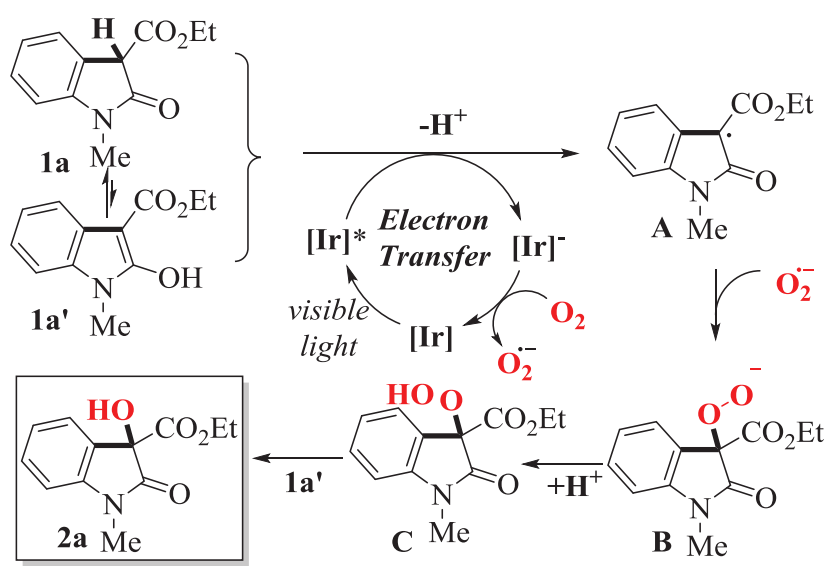

Scheme 2 Proposed mechanism

to the hydroperoxide intermediate $\mathbf{C}$ through intermediate B. Finally, a self-oxidation between $\mathbf{C}$ and $\mathbf{1 a}$ ' furnished the desired product $\mathbf{2 a}$.

\section{Conclusions}

In conclusion, we describe herein a mild photocatalytic system to construct the potentially biologically important molecules 3-ester-3-hydroxy-2-oxindoles in up to 89\% yield. Under the irradiation of visible light, the reaction was carried out open to air at room temperature. Moreover, without the addition of base the optimized conditions were suitable for $\mathrm{N}$-allyl protected and $\mathrm{N}-\mathrm{H}$ free compounds, and the process goes smoothly when enlarged to gram scale. We expect the developed protocol to be useful in the modification of structurally significant chemicals, and further application of this method is ongoing in our laboratory.

\section{Experimental}

\section{General procedure for the photocatalytic reaction}

To a mixture of ethyl 2-oxoindoline-3-carboxylates 1a-1o $(0.5 \mathrm{mmol})$ and $\left[\operatorname{Ir}(\mathrm{ppy})_{2}(\mathrm{dtbbpy})\right] \mathrm{PF}_{6}(2 \mathrm{~mol} \%, 9.1 \mathrm{mg})$ in $\mathrm{CF}_{3} \mathrm{CH}_{2} \mathrm{OH}(5 \mathrm{~mL})$ was added silica gel $(30 \mathrm{mg} / \mathrm{mL})$ at room temperature in a $10 \mathrm{~mL}$ round bottom flask. The resulting solution was stirred at a distance of $5 \mathrm{~cm}$ from an $18 \mathrm{w}$ fluorescent light bulb until the reaction was determined to be completed by TLC analysis. The crude product was purified by silica gel chromatography.

\section{Characterization Data of the Products}

\section{Ethyl 3-hydroxy-1-methyl-2-oxoindoline-3-carboxylate (2a) [10]}

White solid; 88\% yield; m.p. 137 138 ${ }^{\circ} \mathrm{C}$; ${ }^{1} \mathrm{H}$ NMR (400 MHz, $\left.\mathrm{CDCl}_{3}\right): \delta=7.38(\mathrm{t}, J=7.7 \mathrm{~Hz}, 1 \mathrm{H}), 7.29(\mathrm{~d}, J=7.3 \mathrm{~Hz}, 1 \mathrm{H})$, $7.09(\mathrm{t}, J=7.5 \mathrm{~Hz}, 1 \mathrm{H}), 6.87(\mathrm{~d}, J=7.8 \mathrm{~Hz}, 1 \mathrm{H}), 4.61(\mathrm{~s}, 1 \mathrm{H})$, $4.28-4.12$ (m, 2H), 3.23 (s, 3H), $1.15(\mathrm{t}, J=7.1 \mathrm{~Hz}, 3 \mathrm{H}) ;{ }^{13} \mathrm{C}$ NMR (100 MHz, $\left.\mathrm{CDCl}_{3}\right): \delta=173.1,169.8,144.6,130.6,126.9$, 123.7, 123.3, 108.8, 77.3, 63.1, 26.5, 13.8; MS (EI) $m / z$ : 235.1.

\section{Ethyl 3-hydroxy-1,5-dimethyl-2-oxoindoline-3- carboxylate (2b) [10]}

White solid; $77 \%$ yield; m.p. $134 \sim 136{ }^{\circ} \mathrm{C}$; ${ }^{1} \mathrm{H}$ NMR $(400 \mathrm{MHz}$, $\left.\mathrm{CDCl}_{3}\right): \delta=7.17(\mathrm{~d}, J=7.2 \mathrm{~Hz}, 1 \mathrm{H}), 7.10(\mathrm{~s}, 1 \mathrm{H}), 6.76(\mathrm{~d}, J=7.9 \mathrm{~Hz}$, $1 \mathrm{H}), 4.32(\mathrm{~s}, 1 \mathrm{H}), 4.30-4.11(\mathrm{~m}, 2 \mathrm{H}), 3.22(\mathrm{~s}, 3 \mathrm{H}), 2.33$ (s, 3H), 1.17 (t, $J=7.1 \mathrm{~Hz}, 3 \mathrm{H}) ;{ }^{13} \mathrm{C}$ NMR (100 MHz, $\mathrm{CDCl}_{3}$ ): $\delta=173.0,169.8,142.0,132.9,130.7,126.8,124.4,108.5,77.4$, 63.0, 26.5, 20.9, 13.7; MS (EI) m/z: 249.1.

\section{Ethyl 3-hydroxy-5-methoxy-1-methyl-2-oxoindoline- 3-carboxylate (2c)}

Light blue solid; $57 \%$ yield; m.p. $148 \sim 150{ }^{\circ} \mathrm{C}$; ${ }^{1} \mathrm{H}$ NMR $\left(600 \mathrm{MHz}, \mathrm{CDCl}_{3}\right): \delta=6.95-6.86(\mathrm{~m}, 2 \mathrm{H}), 6.84-6.75$ (m, 1H), $4.37(\mathrm{~s}, 1 \mathrm{H}), 4.30-4.15(\mathrm{~m}, 2 \mathrm{H}), 3.79(\mathrm{~s}, 3 \mathrm{H}), 3.22$ (s, 3H), $1.17(\mathrm{t}, J=7.1 \mathrm{~Hz}, 3 \mathrm{H}) ;{ }^{13} \mathrm{C}$ NMR $\left(100 \mathrm{MHz}, \mathrm{CDCl}_{3}\right)$ : 
$\delta=172.8,169.7,156.3,137.8,127.9,115.2,11.5,109.3,77.6$, 63.1, 55.7, 26.6, 13.8; MS (EI) m/z: 265.1; HRMS (ESI): m/z $\left[\mathrm{M}+\mathrm{Na}^{+}\right]$calcd for $\mathrm{C}_{13} \mathrm{H}_{15} \mathrm{NNaO}_{5}: 288.0848$; found: 288.0842 .

\section{Ethyl 3-hydroxy-1-methyl-2-oxo-5-(trifluoromethoxy) indoline-3-carboxylate (2d)}

White solid; 89\% yield; m.p. $77 \sim 79^{\circ} \mathrm{C}$; ${ }^{1} \mathrm{H}$ NMR $(600 \mathrm{MHz}$, $\left.\mathrm{CDCl}_{3}\right): \delta=7.27(\mathrm{~s}, 1 \mathrm{H}), 7.18(\mathrm{~s}, 1 \mathrm{H}), 6.87(\mathrm{~d}, J=8.5 \mathrm{~Hz}, 1 \mathrm{H}), 4.45$ (s, 1H), 4.28-4.17 (m, 2H), 3.25 (s, 3H), $1.17(\mathrm{t}, J=7.1 \mathrm{~Hz}, 3 \mathrm{H})$; ${ }^{13} \mathrm{C}$ NMR $\left(100 \mathrm{MHz}, \mathrm{CDCl}_{3}\right): \delta=173.0,169.1,145.0,143.2$, 128.3, 123.7, $120.4(\mathrm{~d}, J=257.1 \mathrm{~Hz}), 117.8,109.4,77.2,63.4,26.7$, 13.7; ${ }^{19} \mathrm{~F}$ NMR (376 MHz, $\mathrm{CDCl}_{3}$ ): $\delta=-58.34$ (s); MS (EI) $\mathrm{m} / z$ : 319.1; HRMS (EI): $\mathrm{m} / \mathrm{z}\left[\mathrm{M}+\mathrm{NH}_{4}^{+}\right]$calcd for $\mathrm{C}_{13} \mathrm{H}_{16} \mathrm{~F}_{3} \mathrm{~N}_{2} \mathrm{O}_{5}$ : 337.1011; found: 337.1007.

\section{Ethyl 5-fluoro-3-hydroxy-1-methyl-2-oxoindoline-3- carboxylate (2e)}

Light yellow solid; 86\% yield; m.p. $126 \sim 128{ }^{\circ} \mathrm{C}$; ${ }^{1} \mathrm{H}$ NMR $\left(600 \mathrm{MHz}, \mathrm{CDCl}_{3}\right): \delta=7.14-7.06(\mathrm{~m}, 1 \mathrm{H}), 7.04(\mathrm{dd}, J=7.3$, $2.2 \mathrm{~Hz}, 1 \mathrm{H}), 6.81(\mathrm{dd}, J=8.5,3.9 \mathrm{~Hz}, 1 \mathrm{H}), 4.46(\mathrm{~s}, 1 \mathrm{H}), 4.29-$ 4.18 (m, 2H), 3.23 (s, 3H), $1.18(\mathrm{t}, J=7.1 \mathrm{~Hz}, 3 \mathrm{H}) ;{ }^{13} \mathrm{C}$ NMR (100 $\left.\mathrm{MHz}, \mathrm{CDCl}_{3}\right): \delta=172.9(\mathrm{~s}), 169.2(\mathrm{~s}), 159.3(\mathrm{~d}, J=242.6 \mathrm{~Hz}$ ), 140.5 (s), 128.3 (d, $J=8.6 \mathrm{~Hz}), 116.8(\mathrm{~d}, J=23.5 \mathrm{~Hz}), 112.0$ (d, $J=25.4 \mathrm{~Hz}$ ), 109.4 (d, $J=7.9 \mathrm{~Hz}$ ), 77.4 (s), 63.3 (s), 26.7 (s), 13.8 (s). $\left.{ }^{19} \mathrm{~F} \mathrm{NMR} \mathrm{(376} \mathrm{MHz,} \mathrm{CDCl}_{3}\right): \delta=-118.95--119.07$ (m); MS (EI) $m / z: 253.1 ;$ HRMS (EI): $\mathrm{m} / \mathrm{z}\left[\mathrm{M}+\mathrm{H}^{+}\right]$calcd for $\mathrm{C}_{12} \mathrm{H}_{13} \mathrm{FNO}_{4}:$ 254.0829; found: 254.0827 .

\section{Ethyl 5-bromo-3-hydroxy-1-methyl-2-oxoindoline-3- carboxylate (2f)}

White solid; 84\% yield; m.p. 150 153 ${ }^{\circ} \mathrm{C}$; ${ }^{1} \mathrm{H}$ NMR (600 $\left.\mathrm{MHz}, \mathrm{CDCl}_{3}\right): \delta=7.54-7.49(\mathrm{~m}, 1 \mathrm{H}), 7.40(\mathrm{~s}, 1 \mathrm{H}), 6.76$ (d, $J=8.3 \mathrm{~Hz}, 1 \mathrm{H}), 4.38(\mathrm{~s}, 1 \mathrm{H}), 4.31-4.16(\mathrm{~m}, 2 \mathrm{H}), 3.22$ (s, 3H), 1.19 (t, $J=7.1 \mathrm{~Hz}, 3 \mathrm{H}) ;{ }^{13} \mathrm{C}$ NMR $\left(100 \mathrm{MHz}, \mathrm{CDCl}_{3}\right)$ : $\delta=172.6,169.1,143.6,133.4,128.7,127.0,115.8,110.3,77.1$, 63.4, 26.7, 13.8; MS (EI) $m / z$ : 313.0; HRMS (EI): $\mathrm{m} / \mathrm{z}\left[\mathrm{M}+\mathrm{H}^{+}\right]$ calcd for $\mathrm{C}_{12} \mathrm{H}_{13} \mathrm{BrNO}_{4}: 314.0028$; found: 314.0022 .

\section{Ethyl 6-bromo-3-hydroxy-1-methyl-2-oxoindoline-3- carboxylate (2g)}

Light blue solid; $20 \%$ yield; m.p. $155 \sim 156{ }^{\circ} \mathrm{C}$; ${ }^{1} \mathrm{H}$ NMR $\left(400 \mathrm{MHz}, \mathrm{CDCl}_{3}\right): \delta=7.23(\mathrm{~s}, 1 \mathrm{H}), 7.14(\mathrm{~d}, J=7.8 \mathrm{~Hz}, 1 \mathrm{H})$, 7.03 (s, 1H), 4.34 (s, 1H), $4.28-4.16(\mathrm{~m}, 2 \mathrm{H}), 3.22(\mathrm{~s}, 3 \mathrm{H})$, $1.17(\mathrm{t}, J=7.1 \mathrm{~Hz}, 3 \mathrm{H}) ;{ }^{13} \mathrm{C} \mathrm{NMR}\left(100 \mathrm{MHz}, \mathrm{CDCl}_{3}\right): \delta=172.9$, 169.2, 145.8, 126.1, 125.8, 125.0, 124.3, 112.4, 76.9, 63.4, 26.7, 13.8; MS (EI) $m / z: 313.0$; HRMS (EI): $\mathrm{m} / \mathrm{z}\left[\mathrm{M}+\mathrm{H}^{+}\right]$calcd for $\mathrm{C}_{12} \mathrm{H}_{13} \mathrm{BrNO}_{4}: 314.0028$; found: 314.0024 .

\section{Ethyl 7-fluoro-3-hydroxy-1-methyl-2-oxoindoline-3- carboxylate (2i)}

White solid; 79\% yield; m.p. $118 \sim 119{ }^{\circ} \mathrm{C}$; ${ }^{1} \mathrm{H}$ NMR (400 $\left.\mathrm{MHz}, \mathrm{CDCl}_{3}\right): \delta=7.20-6.98(\mathrm{~m}, 3 \mathrm{H}), 4.38(\mathrm{~s}, 1 \mathrm{H}), 4.34-$ 4.15 (m, 2H), 3.45 (s, 3H), $1.18(\mathrm{t}, J=7.0 \mathrm{~Hz}, 3 \mathrm{H}) ;{ }^{13} \mathrm{C}$ NMR $\left(100 \mathrm{MHz}, \mathrm{CDCl}_{3}\right): \delta=172.7(\mathrm{~s}), 169.2(\mathrm{~s}), 147.6(\mathrm{~d}, J=244.6$ $\mathrm{Hz}), 131.1(\mathrm{~d}, J=8.9 \mathrm{~Hz}), 129.5(\mathrm{~s}), 124.0(\mathrm{~d}, J=6.1 \mathrm{~Hz}), 119.6$ (s), $118.6(\mathrm{~d}, J=19.2 \mathrm{~Hz}), 77.2(\mathrm{~s}), 63.3(\mathrm{~s}), 29.1(\mathrm{~s}), 13.8(\mathrm{~s})$; ${ }^{19} \mathrm{~F}$ NMR (376 MHz, $\mathrm{CDCl}_{3}$ ): $\delta=-135.27--135.69$ (m); MS (EI) $\mathrm{m} / z: 253.1 ; \mathrm{HRMS}$ (EI): $\mathrm{m} / \mathrm{z}\left[\mathrm{M}+\mathrm{H}^{+}\right]$calcd for $\mathrm{C}_{12} \mathrm{H}_{13} \mathrm{FNO}_{4}$ : 254.0829; found: 254.0820 .

\section{Ethyl 3-hydroxy-2-oxoindoline-3-carboxylate (2j)}

White solid; $82 \%$ yield; m.p. $147 \sim 148{ }^{\circ} \mathrm{C}$; ${ }^{1} \mathrm{H}$ NMR (600 $\left.\mathrm{MHz}, \mathrm{CDCl}_{3}\right): \delta=8.47(\mathrm{~s}, 1 \mathrm{H}), 7.32(\mathrm{t}, J=7.7 \mathrm{~Hz}, 1 \mathrm{H}), 7.28$ $(\mathrm{s}, 1 \mathrm{H}), 7.08(\mathrm{t}, J=7.5 \mathrm{~Hz}, 1 \mathrm{H}), 6.94(\mathrm{~d}, J=7.7 \mathrm{~Hz}, 1 \mathrm{H})$, $4.51(\mathrm{~s}, 1 \mathrm{H}), 4.31-4.14(\mathrm{~m}, 2 \mathrm{H}), 1.18(\mathrm{t}, J=7.1 \mathrm{~Hz}, 3 \mathrm{H})$; ${ }^{13} \mathrm{C}$ NMR (100 MHz, DMSO-d $\left.\mathrm{d}^{6}\right): \delta=174.7,169.3,142.8$, 130.3, 129.2, 123.9, 122.1, 110.2, 77.6, 61.3, 14.0; MS (EI) $\mathrm{m} / z$ : 221.1; HRMS (EI): $\mathrm{m} / \mathrm{z}\left[\mathrm{M}+\mathrm{H}^{+}\right]$calcd for $\mathrm{C}_{11} \mathrm{H}_{12} \mathrm{NO}_{4}$ : 222.0766; found: 222.0762 .

\section{Ethyl 1-benzyl-3-hydroxy-2-oxoindoline-3-carboxylate (2k) [10]}

Light blue solid; $79 \%$ yield; m.p. $140 \sim 143{ }^{\circ} \mathrm{C}$; ${ }^{1} \mathrm{H}$ NMR (400 $\left.\mathrm{MHz}, \mathrm{CDCl}_{3}\right): \delta=7.46-7.27(\mathrm{~m}, 7 \mathrm{H}), 7.06(\mathrm{t}, J=7.6 \mathrm{~Hz}$, $1 \mathrm{H}), 6.71(\mathrm{~d}, J=7.6 \mathrm{~Hz}, 1 \mathrm{H}), 5.19(\mathrm{~d}, J=15.9 \mathrm{~Hz}, 1 \mathrm{H}), 4.68$ $(\mathrm{d}, J=16.0 \mathrm{~Hz}, 1 \mathrm{H}), 4.39(\mathrm{~s}, 1 \mathrm{H}), 4.36-4.26(\mathrm{~m}, 1 \mathrm{H}), 4.24-4.08$ (m, $1 \mathrm{H}), 1.20$ (t, $J=7.1 \mathrm{~Hz}, 3 \mathrm{H}) ;{ }^{13} \mathrm{C}$ NMR $\left(100 \mathrm{MHz}, \mathrm{CDCl}_{3}\right)$ : $\delta=173.3,169.7,143.5,135.0,130.5,128.6,127.6,126.9,123.7$, 123.3, 109.8, 77.4, 63.2, 43.7, 13.7; MS (EI) $m / z: 311.1$.

\section{Ethyl 3-hydroxy-1-(4-methoxybenzyl)-2-oxoindoline- 3-carboxylate (2l)}

Light blue solid; 74\% yield; m.p. 131 132 ${ }^{\circ} \mathrm{C}$; ${ }^{1} \mathrm{H}$ NMR $\left(600 \mathrm{MHz}, \mathrm{CDCl}_{3}\right): \delta=7.28(\mathrm{~d}, J=7.5 \mathrm{~Hz}, 1 \mathrm{H}), 7.25$ $(\mathrm{d}, J=8.4 \mathrm{~Hz}, 3 \mathrm{H}), 7.05(\mathrm{t}, J=7.6 \mathrm{~Hz}, 1 \mathrm{H}), 6.84(\mathrm{~d}, J=8.6 \mathrm{~Hz}$, 2H), $6.73(\mathrm{~d}, J=7.9 \mathrm{~Hz}, 1 \mathrm{H}), 5.10(\mathrm{~d}, J=15.6 \mathrm{~Hz}, 1 \mathrm{H}), 4.63$ 
$(\mathrm{d}, J=15.6 \mathrm{~Hz}, 1 \mathrm{H}), 4.37(\mathrm{~s}, 1 \mathrm{H}), 4.34-4.27(\mathrm{~m}, 1 \mathrm{H})$, $4.22-4.12(\mathrm{~m}, 1 \mathrm{H}), 3.78(\mathrm{~s}, 3 \mathrm{H}), 1.19(\mathrm{t}, J=7.1 \mathrm{~Hz}, 3 \mathrm{H}) ;{ }^{13} \mathrm{C}$ $\operatorname{NMR}\left(100 \mathrm{MHz}_{1} \mathrm{CDCl}_{3}\right)$ : $\delta=173.2,169.7,158.9,143.5,130.4$, 128.3, 126.9, 126.9, 123.6, 123.2, 114.0, 109.8, 77.4, 63.1, 55.1, 43.2, 13.7; MS (EI) $m / z: 341.1$; HRMS (ESI): $\mathrm{m} / \mathrm{z}\left[\mathrm{M}+\mathrm{Na}^{+}\right]$ calcd for $\mathrm{C}_{19} \mathrm{H}_{19} \mathrm{NNaO}_{5}$ : 364.1161; found: 364.1155 .

\section{Ethyl 1-allyl-3-hydroxy-2-oxoindoline-3-carboxylate (2m)}

White solid; 39\% yield; m.p. 136 137 ${ }^{\circ} \mathrm{C}$; ${ }^{1} \mathrm{H}$ NMR $(600$ $\left.\mathrm{MHz}, \mathrm{CDCl}_{3}\right): \delta=7.34$ (t, $\left.J=7.8 \mathrm{~Hz}, 1 \mathrm{H}\right), 7.29(\mathrm{~d}, J=7.4 \mathrm{~Hz}$, 1H), 7.08 (t, $J=7.5 \mathrm{~Hz}, 1 \mathrm{H}), 6.84(\mathrm{~d}, J=7.8 \mathrm{~Hz}, 1 \mathrm{H}), 5.90-$ $5.80(\mathrm{~m}, 1 \mathrm{H}), 5.29-5.18(\mathrm{~m}, 2 \mathrm{H}), 4.49(\mathrm{~d}, J=16.8 \mathrm{~Hz}, 1 \mathrm{H})$, $4.32-4.12(\mathrm{~m}, 4 \mathrm{H}), 1.16(\mathrm{t}, J=7.1 \mathrm{~Hz}, 3 \mathrm{H}) ;{ }^{13} \mathrm{C}$ NMR $(100$ $\left.\mathrm{MHz}, \mathrm{CDCl}_{3}\right): \delta=172.9,169.7,143.6,130.5,130.4,126.8$, 123.7, 123.3, 117.1, 109.6, 77.4, 63.2, 42.3, 13.7; MS (EI) $m / z$ : 261.1; HRMS (ESI): $\mathrm{m} / \mathrm{z}\left[\mathrm{M}+\mathrm{Na}^{+}\right]$calcd for $\mathrm{C}_{14} \mathrm{H}_{15} \mathrm{NNaO}_{4}$ : 284.0899; found: 284.0893 .

\section{Isobutyl 3-hydroxy-1-methyl-2-oxoindoline-3- carboxylate (2n)}

Light blue solid; 77\% yield; m.p. 70 72 ${ }^{\circ} \mathrm{C} ;{ }^{1} \mathrm{H}$ NMR (600 $\left.\mathrm{MHz}, \mathrm{CDCl}_{3}\right): \delta=7.38(\mathrm{t}, J=7.8 \mathrm{~Hz}, 1 \mathrm{H}), 7.28(\mathrm{~d}, J=7.4$ $\mathrm{Hz}, 1 \mathrm{H}), 7.09$ (t, $J=7.5 \mathrm{~Hz}, 1 \mathrm{H}), 6.88$ (d, $J=7.8 \mathrm{~Hz}, 1 \mathrm{H}), 4.32$ $(\mathrm{s}, 1 \mathrm{H}), 3.94(\mathrm{p}, J=10.5 \mathrm{~Hz}, 2 \mathrm{H}), 3.24(\mathrm{~s}, 3 \mathrm{H}), 1.87-1.75(\mathrm{~m}, 1 \mathrm{H})$, $0.72(\mathrm{t}, J=6.7 \mathrm{~Hz}, 6 \mathrm{H}) ;{ }^{13} \mathrm{C} \mathrm{NMR}\left(100 \mathrm{MHz}, \mathrm{CDCl}_{3}\right): \delta=173.1$, 169.7, 144.4, 130.5, 127.0, 123.6, 123.2, 108.7, 77.4, 72.4, 27.5, 26.5, 18.4; MS (EI) $m / z: 263.1$; HRMS (ESI): $\mathrm{m} / \mathrm{z}\left[\mathrm{M}+\mathrm{Na}^{+}\right]$ calcd for $\mathrm{C}_{14} \mathrm{H}_{17} \mathrm{NNaO}_{4}$ : 286.1055; found: 286.1050 .

\section{Benzyl 3-hydroxy-1-methyl-2-oxoindoline- 3-carboxylate (20)}

Light blue solid; 62\% yield; m.p. $116 \sim 118{ }^{\circ} \mathrm{C} ;{ }^{1} \mathrm{H}$ NMR $\left(600 \mathrm{MHz}, \mathrm{CDCl}_{3}\right): \delta=7.38(\mathrm{t}, J=7.7 \mathrm{~Hz}, 1 \mathrm{H}), 7.29-7.26$ (m, 3H), 7.24 (d, $J=7.4 \mathrm{~Hz}, 1 \mathrm{H}), 7.13-7.03$ (m, 3H), 6.87 $(\mathrm{d}, J=7.8 \mathrm{~Hz}, 1 \mathrm{H}), 5.19$ (q, $J=12.5 \mathrm{~Hz}, 2 \mathrm{H}), 4.29(\mathrm{~s}, 1 \mathrm{H})$, 3.23 (s, 3H); ${ }^{13} \mathrm{C}$ NMR (100 MHz, $\left.\mathrm{CDCl}_{3}\right): \delta=173.0,169.5$, 144.4, 134.6, 130.7, 128.4, 128.2, 127.2, 126.7, 123.8, 123.3, 108.8, 77.5, 68.0, 26.6; MS (ESI) $m / z$ : 297.1; HRMS (ESI): $\mathrm{m} / \mathrm{z}\left[\mathrm{M}+\mathrm{Na}^{+}\right]$calcd for $\mathrm{C}_{17} \mathrm{H}_{15} \mathrm{NNaO}_{4}: 320.0899$; found: 320.0893.

\section{3-Hydroxy-3-phenylindolin-2-one (2p) [33]}

White solid; 60\% yield; ${ }^{1} \mathrm{H}$ NMR (500 MHz, DMSO-d ${ }^{6}$ ): $\delta=10.41(\mathrm{~s}, 1 \mathrm{H}), 7.34-7.22(\mathrm{~m}, 6 \mathrm{H}), 7.11(\mathrm{~d}, J=7.3 \mathrm{~Hz}, 1 \mathrm{H})$, $6.97(\mathrm{t}, J=7.5 \mathrm{~Hz}, 1 \mathrm{H}), 6.91(\mathrm{~d}, J=7.7 \mathrm{~Hz}, 1 \mathrm{H}), 6.67-6.61$

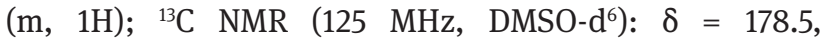
$141.9,141.5,133.7,129.2,128.0,127.4,125.4,124.8,122.0$, 109.8, 77.3 .

\section{3-Hydroxy-1-methyl-3-phenylindolin-2-one (2q) [33]}

White solid; $17 \%$ yield; ${ }^{1} \mathrm{H}$ NMR (500 MHz, DMSO- $\mathrm{d}^{6}$ ): $\delta=7.35(\mathrm{t}, J=7.7 \mathrm{~Hz}, 1 \mathrm{H}), 7.32-7.24(\mathrm{~m}, 5 \mathrm{H}), 7.14$ $(\mathrm{d}, J=7.2 \mathrm{~Hz}, 1 \mathrm{H}), 7.09(\mathrm{~d}, J=7.8 \mathrm{~Hz}, 1 \mathrm{H}), 7.05(\mathrm{t}, J=7.5 \mathrm{~Hz}, 1 \mathrm{H})$, 6.69 (s, 1H), 3.16 (s, 3H); ${ }^{13} \mathrm{C}$ NMR (125 MHz, DMSO-d $\left.\mathrm{d}^{6}\right)$ : $\delta=176.7,143.4,141.3,133.0,129.4,128.1,127.5,125.5,124.3$, 122.7, 108.8, 77.0, 26.1 .

Acknowledgements: We are grateful to the National Natural Science Foundation of China (No. 21862018), and Tarim University (TDZKJC201701) for financial support for this research.

Conflict of interest: The authors state no conflict of interest.

\section{References}

[1] Peddibhotla S. 3-Substituted-3-hydroxy-2-oxindole, an emerging new scaffold for drug discovery with potential anti-cancer and other biological Activities. Curr Bioact Compd. 2009;5(1):20-38.

[2] Zhou F, Liu YL, Zhou J. Catalytic asymmetric synthesis of oxindoles bearing a tetrasubstituted stereocenter at the C-3 position. Adv Synth Catal. 2010;352(9):1381-407.

[3] Kamano Y, Zhang HP, Ichihara Y, Kizu H, Komiyama K, Pettit GR. Convolutamydine $\mathrm{A}$, a novel bioactive hydroxyoxindole alkaloid from marine bryozoan Amathia convoluta. Tetrahedron Lett. 1995;36(16):2783-4.

[4] Takao T, Kitatani F, Watanabe N, Yagi A, Sakata K. A simple screening method for antioxidants and isolation of several antioxidants produced by marine bacteria from fish and shellfish. Biosci Biotechnol Biochem. 1994;58(10):1780-3.

[5] Komakine N, Takaishi Y, Honda G, Ito M, Takeda Y, Kodzhimatov OK, et al. Indole alkaloids from Rheum maximowiczii. Nat Med. 2005;59(1):45-8.

[6] Bergonzini G, Melchiorre P. Dioxindole in asymmetric catalytic synthesis: routes to enantioenriched 3-substituted 3-hydroxyoxindoles and the preparation of maremycin A. Angew Chem Int Ed Engl. 2012;51(4):971-4.

[7] Ghosh S, Kinthada LK, Bhunia S, Bisai A. Lewis acid-catalyzed Friedel-Crafts alkylations of 3-Hydroxy-2-oxindole: an efficient approach to the core structure of azonazine. Chem Commun (Camb). 2012;48(81):10132-4.

[8] Song L, Guo QX, Li XC, Tian J, Peng YG. The direct asymmetric $\alpha$ alkylation of ketones by Brønsted acid catalysis. Angew Chem Int Ed Engl. 2012;51(8):1899-902. 
[9] Yin X, Xu P, Dong K, Liao K, Zhou F, Zhou J. Ga(OTf) ${ }_{3}$ Catalyzed highly efficient substitution reaction of 3-hydroxyoxindoles using TMSN $_{3}$. Acta Chimi Sin. 2015;73(7):685-9.

[10] Wang J, Yuan Y, Xiong R, Zhang-Negrerie D, Du Y, Zhao K. Phenyliodine bis(trifluoroacetate)-mediated oxidative C-C bond formation: synthesis of 3-hydroxy-2-oxindoles and spirooxindoles from anilides. Org Lett. 2012;14(9): 2210-3.

[11] Liu Y, Zhu F, Wang C, Zhou J. Metal catalysis versus organocatalysis in the catalytic asymmetric synthesis of 3-hydroxyoxindole. Youji Huaxue. 2013;33(8):1595-615.

[12] Brandão P, Burke AJ. Recent advances in the asymmetric catalytic synthesis of chiral 3-hydroxy and 3-aminooxindoles and derivatives: medicinally relevant compounds. Tetrahedron. 2018;74(38):4927-57.

[13] Tomita D, Yamatsugu K, Kanai M, Shibasaki M. Enantioselective synthesis of SM-130686 based on the development of asymmetric $\mathrm{Cu}(\mathrm{I}) \mathrm{F}$ catalysis to access 2-oxindoles containing a tetrasubstituted carbon. J Am Chem Soc. 2009;131(20):6946-8.

[14] Jia YX, Katayev D, Kündig EP. Synthesis of 3-hydroxyoxindoles by Pd-catalysed intramolecular nucleophilic addition of aryl halides to $\alpha$-ketoamides. Chem Commun (Camb). 2010;46(1):130-2.

[15] Yin L, Kanai M, Shibasaki M. A facile pathway to enantiomerically enriched 3-hydroxy-2-oxindoles: asymmetric intramolecular arylation of $\alpha$-keto amides catalyzed by a palladium-difluorphos complex. Angew Chem Int Ed Engl. 2011;50(33):7620-3.

[16] Guo X, Huang H, Yang L, Hu W. Trapping of oxonium ylide with isatins: efficient and stereoselective construction of adjacent quaternary carbon centers. Org Lett. 2007;9(23): 4721-3.

[17] Itoh J, Han SB, Krische MJ. Enantioselective allylation, crotylation, and reverse prenylation of substituted isatins: iridium-catalyzed C-C bond-forming transfer hydrogenation. Angew Chem Int Ed Engl. 2009;48(34):6313-6.

[18] Liu YL, Wang BL, Cao JJ, Chen L, Zhang YX, Wang C, et al. Organocatalytic asymmetric synthesis of substituted 3-hydroxy-2-oxindoles via Morita-Baylis-Hillman reaction. J Am Chem Soc. 2010;132(43):15176-8.

[19] Fu XP, Liu L, Wang D, Chen YJ, Li CJ. “On water”-promoted direct alkynylation of isatins catalyzed by NHC-silver complexes for the efficient synthesis of 3-hydroxy-3-ethynylindolin-2-ones. Green Chem. 2011;13(3):549-53.

[20] Gutierrez EG, Wong CJ, Sahin AH, Franz AK. Enantioselective and regioselective indium(III)-catalyzed addition of pyrroles to isatins. Org Lett. 2011;13(21):5754-7.

[21] Liu Z, Gu P, Shi M, McDowell P, Li G. Catalytic asymmetric addition of arylboronic acids to isatins using $\mathrm{C}_{2}$-symmetric cationic $\mathrm{N}$-heterocyclic carbenes (NHCs) $\mathrm{Pd}^{2+}$ diaqua complexes as catalysts. Org Lett. 2011;13(9):2314-7.

[22] Zheng K, Yin C, Liu X, Lin L, Feng X. Catalytic Asymmetric addition of alkyl enol ethers to 1,2-dicarbonyl compounds: highly enantioselective synthesis of substituted 3-alkyl-3-hydroxyoxindoles. Angew Chem Int Ed Engl. 2011;50(11):2573-7.

[23] Niu R, Xiao J, Liang T, Li X. Facile synthesis of azaarenesubstituted 3-hydroxy-2-oxindoles via Brønsted acid catalyzed $\mathrm{sp}^{3} \mathrm{C}$-H functionalization. Org Lett. 2012;14(3):676-9.
[24] Zhong F, Yao W, Dou X, Lu Y. Enantioselective construction of 3-hydroxy oxindoles via decarboxylative addition of $\beta$-ketoacids to isatins. Org Lett. 2012;14(15):4018-21.

[25] Wu Z, Fang X, Leng Y, Yao H, Lin A. Indium-mediated palladiumcatalyzed allylic alkylation of isatins with alkynes. Adv Synth Catal. 2018;360(6):1289-95.

[26] Zhang YY, Chen H, Jiang X, Liang H, He X, Zhang Y, et al. Nickel(II)-catalyzed addition reaction of arylboronic acids to isatins. Tetrahedron. 2018;74(18):2245-50.

[27] Laina-Martín V, Humbrías-Martín J, Fernández-Salas JA, Alemán J. Asymmetric vinylogous Mukaiyama aldol reaction of isatins under bifunctional organocatalysis: enantioselective synthesis of substituted 3-hydroxy-2-oxindoles. Chem Commun (Camb). 2018;54(22):2781-4.

[28] Ishimaru T, Shibata N, Nagai J, Nakamura S, Toru T, Kanemasa S. Lewis acid-catalyzed enantioselective hydroxylation reactions of oxindoles and $\beta$-keto esters using DBFOX ligand. J Am Chem Soc. 2006;128(51):16488-9.

[29] Sano D, Nagata K, Itoh T. Catalytic asymmetric hydroxylation of oxindoles by molecular oxygen using a phase-transfer catalyst. Org Lett. 2008;10(8):1593-5.

[30] Bui T, Candeias NR, Barbas CF 3rd. Dimeric Quinidine-catalyzed enantioselective amino oxygenation of oxindoles: an organocatalytic approach to 3-hydroxyoxindole derivatives. J Am Chem Soc. 2010;132(16):5574-5.

[31] Liao YH, Wu Z], Han WY, Zhang XM, Yuan WC. Organocatalytic enantioselective stereoablative hydroxylation of 3-halooxindoles: an effective method for the construction of enantioenriched 3-substituted 3-hydroxy-2-oxindoles. Chemistry. 2012;18(29):8916-20.

[32] Yang Y, Moinodeen F, Chin W, Ma T, Jiang Z, Tan CH. Pentanidium-catalyzed enantioselective $\alpha$-hydroxylation of oxindoles using molecular oxygen. Org Lett. 2012;14(18):4762-5.

[33] Zhu WM, Bao WH, Ying WW, Chen WT, Huang YL, Ge GP, et al. TEMPO-promoted $\mathrm{C}\left(\mathrm{sp}^{3}\right)-\mathrm{H}$ hydroxylation of 2-oxindoles at room temperature. Asian J Org Chem. 2018;7(2):337-40.

[34] Narayanam JM, Stephenson CR. Visible light photoredox catalysis: applications in organic synthesis. Chem Soc Rev. 2011;40(1):102-13.

[35] Xuan J, Xiao W]. Visible-light photoredox catalysis. Angew Chem Int Ed Engl. 2012;51(28):6828-38.

[36] Prier CK, Rankic DA, MacMillan DW. Visible light photoredox catalysis with transition metal complexes: applications in organic synthesis. Chem Rev. 2013;113(7):5322-63.

[37] Schultz DM, Yoon TP. Solar synthesis: prospects in visible light photocatalysis. Science. 2014;343(6174):1239176.

[38] Romero NA, Nicewicz DA. Organic photoredox catalysis. Chem Rev. 2016; 116:10075-166.

[39] Skubi KL, Blum TR, Yoon TP. Dual catalysis strategies in photochemical synthesis. Chem Rev. 2016;116(17):10035-74.

[40] Ghogare AA, Greer A. Using singlet oxygen to synthesize natural products and drugs. Chem Rev. 2016;116(17): 9994-10034.

[41] Sundén H, Engqvist M, Casas J, Ibrahem I, Córdova A. Direct amino acid catalyzed asymmetric $\alpha$ oxidation of ketones with molecular oxygen. Angew Chem Int Ed Engl. 2004;43(47):6532-5.

[42] Córdova A, Sundén H, Engqvist M, Ibrahem I, Casas J. The direct amino acid-catalyzed asymmetric incorporation of 
molecular oxygen to organic compounds. J Am Chem Soc. 2004;126(29):8914-5.

[43] Lian M, Li Z, Cai Y, Meng Q, Gao Z. Enantioselective photooxygenation of $\beta$-keto esters by chiral phase-transfer catalysis using molecular oxygen. Chem Asian J. 2012;7(9):2019-23.

[44] Ding W, Lu LQ, Zhou QQ, Wei Y, Chen JR, Xiao WJ. Bifunctional photocatalysts for enantioselective aerobic oxidation of $\beta$-ketoesters. J Am Chem Soc. 2017;139(1):63-6.

[45] Xia XD, Ren YL, Chen JR, Yu XL, Lu LQ, Zou YQ, et al. Phototandem catalysis: efficient synthesis of 3-ester-3- hydroxy-2-oxindoles by a visible light-induced cyclization of diazoamides through an aerobic oxidation sequence. Chem Asian J. 2015;10(1):124-8.

[46] Maity S, Zheng N. A Visible-light-mediated oxidative C-N bond formation/aromatization cascade: photocatalytic preparation of N-arylindoles. Angew Chem Int Ed Engl. 2012;51(38): 9562-6.

[47] Shuklov I, Dubrovina N, Börner A. Fluorinated alcohols as solvents, cosolvents and additives in homogeneous catalysis. Synthesis. 2007;2007(19):2925-43. 